\title{
Study on Psychological Influence of Nursing Intervention on Orthopedic Operation Patients during Perioperative Period
}

\author{
Zeng Rong* \\ The People's Hospital of Longquanyi District, Chengdu, Sichuan 610100, China
}

\begin{abstract}
Objective: to discuss psychological influence of orthopedic operation patients with nursing intervention during perioperative period. Methods: 106 cases of orthopedic operation patients from August 2014 to March 2015 in our hospital were selected. According to the random number table, all cases were divided into two groups with principle of informed consent. The control group: 53 cases were treated with the conventional nursing; the intervention group: 53 cases were treated with the nursing intervention. Psychological reactions were compared in the two groups. Results: After nursing, the intervention group had the good rate of psychological reaction $86.8 \%$, which was much higher than that in the control group (66.0\%). Different results between the two groups were significant in Statistics $(p<0.05)$; however, the intervention group had the rate of pain occurrence $54.7 \%$, which was much lower than that in the control group (73.6\%). Different results between the two groups were significant in Statistics $(p<0.05)$. Conclusion: Nursing intervention could obviously improve psychological state of patients, reduce pain occurrence and accelerate recovery.
\end{abstract}

\author{
KEYWORDS \\ Orthopaedics \\ Psychological reaction \\ Nursing intervention
}

\section{Introduction}

With the development and progress of technology, orthopaedics makes a big progress in the diagnosis and treatment and the surgical treatment is one of the main treatments of orthopedic diseases [1]. Nursing on orthopedic operation patients during perioperative period should draw attention. Facing operation, common patients have certain psychological matters. So nursing measures on psychological influence of patients during perioperative period are directly related to therapeutic effect and rehabilitation condition. With the study on psychological influence of orthopedic operation patients with nursing intervention during perioperative period, the author summarized and

Copyright $\odot 2015$ Zeng Rong

doi: $10.18686 /$ jn.v4i2.4

Received: February 21, 2015; Accepted: May 7, 2015; Published online: June 27, 2015

This is an open-access article distributed under the terms of the Creative Commons Attribution Unported License (http://creativecommons.org/ licenses/by-nc/4.0/), which permits unrestricted use, distribution, and reproduction in any medium, provided the original work is properly cited.

${ }^{\star}$ Corresponding author: The People's Hospital of Longquanyi District, Chengdu City, Sichuan Province 610100, China. E-mail: rong z2012@163.com analyzed the related data for providing reference information in clinical nursing.

\section{Data and methods 2.1. General data}

106 cases of orthopedic operation patients from August 2014 to March 2015 in our hospital were selected. 106 cases in total with 66 men and 40 women from $20-73$ years old (mean age $41.4 \pm 3.3$ ), in which 61 cases with limb fracture, 17 cases with hip fracture, 9 cases with spinal fractures and 19 cases with other fractures. According to the random number table, all cases were divided into 53 cases in the control group and 53 cases in the intervention group with principle of informed consent. Different results between the two groups were not significant in statistics $(p>$ $0.05)$, so general data could be compared. See Table 1.

\subsection{Method}

Patients in the control group are treated with the conventional nursing. (1) Conventional skin preparation before operation. Shave skin hair on operation area completely, then clean and dry. Use iodine tincture to smear, and then use $75 \%$ ethanol in the deiodination or benzalkonium bromide to smear the area on the day before operation day or 
Table 1. General data comparison between two groups $(n=53)$.

\begin{tabular}{ccccccc}
\hline \multirow{2}{*}{ Group } & \multirow{2}{*}{ Male/Female } & Age & \multicolumn{3}{c}{ Injury parts } \\
\cline { 5 - 7 } & & & Lip fracture & Hip fracture & Spinal fracture & Other fracture \\
\hline Intervention group & $34 / 19$ & $41.5 \pm 3.2$ & 31 & 9 & 4 & 9 \\
Control group & $32 / 21$ & $41.2 \pm 3.0$ & 30 & 8 & 5 & 10 \\
\hline
\end{tabular}

Note: Compared with the control group, $p>0.05$ ( $x^{2}$ test, $t$ test).

on the operation day once. (2) After operation, elevate the affected limb by using stents, pillows and sandbags, etc, for blood return. Fixed limb with gypsum is put comfortably for better venous drainage and preventing gypsum fracture and compression of local soft tissue. (3) Accompanying persons should turn over patients lying in bed regularly to keep local blood circulation unobstructed. What's more, patients lying in bed must notice the cleaning and health. Urinary and fecal incontinence, puckery bed sheets and clastics on the bed can cause skin resistance decreased and bedsore. Encourage patients to drink more water and eat more digestible food for normal defecation,avoid constipation and prevent malnutrition. Encourage patients to do exercises on the bed in early times, such as pulling rings and evaluating body to increase vital capacity. When discharge, tell family members to clean the patient's room absolutely, put away all unneeded furniture and sundries to give the patient enough room for walking. Pave antiskid pad in the toilet and install handrails and lamps to prevent falling. It's continent for the patient to use the toilet under some light at night [2].

Patients in the intervention group are treated with nursing intervention on the basis of the control group. (1) Psychological intervention: Most patients are nervous and anxious about the effect of the treatment efficacy on daily life quality. Before operation, nursing staff should explain the good prognosis, which makes patients a calm mood and an active psychology to face the surgical treatment. After operation, communicate with patients after daily ward round, which is good for recovery [3]. (2) Behavior intervention: Strengthen rehabilitation guidance for patients and guide patients to recover function exercise in time, in order to recover local limb function and health in whole body and prevent complications, which can achieve the expected effect. In the early stage, help patients to do exercises. The active value was changes from light to heavy and the active extent was changes from small to big. In the middle stage, according to the disease, increase amount of exercise, strength and time combined with easy mechanical auxiliary or stent auxiliary. In the late stage, strengthen exercise to the disease in order to recover limb function as soon as possible.

\subsection{Observation contents and judgment criteria}

The grades of psychological reaction: Grade I refers to stable and calm emotion, Grade II refers to a little nervous but can control by oneself, Grade III refers to nervous and anxious, limbs with light shaking and Grade IV refers to very nervous, whole body shaking and out of control. The rate of good reaction adds Grade I and Grade II.

Visual Analogue Scale (VAS): 0 3 mild degree, 4 6 moderate degree, $7 \sim 10$ severe degree. The rate of pain occurrence adds the moderate degree and severe degree.

\subsection{Statistical processing}

Data in research are calculated into the SPSS 13.0 software to complete the analysis, measurement data is represented by mean \pm standard deviation $(\bar{x} \pm s)$ using $t$-test, count data using $x^{2}$ test, $p<0.05$. Different results were significant in Statistics.

\section{Results}

After nursing, the intervention group had the good rate of psychological reaction $86.8 \%$, which was much higher than that in the control group (66.0\%). Different results between the two groups were significant in Statistics $(p<$ $0.05)$; however, the intervention group had the rate of pain occurrence $54.7 \%$, which was much lower than that in the

Table 2. Psychological reaction comparison between two groups $(n=53)$.

\begin{tabular}{|c|c|c|c|c|c|}
\hline Group & Grade I & Grade II & Grade III & Grade IV & Good Reaction \\
\hline Intervention Group & $15(28.3)$ & $31(58.5)$ & $6(11.3)$ & $1(1.9)$ & $46(86.8)^{*}$ \\
\hline Control Group & $9(17.0)$ & $26(49.1)$ & $12(22.6)$ & $6(11.3)$ & $35(66.0)$ \\
\hline
\end{tabular}

Note: Compared with the control group, ${ }^{*} p<0.05$ ( $x^{2}$ test).

Table 3. Pain occurrence comparison between two groups $(n=53)$.

\begin{tabular}{ccccc} 
Group & Mild Degree & Moderate Degree & Severe Degree & Pain \\
\hline Intervention Group & $24(43.5)$ & $27(50.9)$ & $2(3.8)$ & $29(54.7)^{\star}$ \\
Control Group & $14(26.4)$ & $25(47.2)$ & $14(26.4)$ & $39(73.6)$ \\
\hline
\end{tabular}

Note: Compared with the control group, ${ }^{*} p<0.05$ ( $x^{2}$ test). 
control group (73.6\%). Different results between the two groups were significant in Statistics $(p<0.05)$. See Table 2 and 3.

\section{Discussion}

\subsection{Great importance attached to orthopedic nursing}

With the development of modern society, transportation and industry develop rapidly. Orthopedic patients become more and more, surgical treatment is one of the main treatment methods [4-6]. Because most patients have fear and anxiety in different levels and worry about postoperative prognosis. They are afraid of bad recover causing the loss of life and work ability and affecting daily life quality. So nursing on orthopedic patients should be paid more attention too.

\subsection{Nursing state during orthopedic perioperative period}

Nursing work during perioperative period has been relatively standardized. Patients in the control group are treated with the conventional nursing, and they know the disease and operation plans, which reduce their fear and tensity. They can take the operation with the best psychological state. According to the disease, choose people to accompany, ask patients to relax on hard bed, give analgesics to patients with severe pain, examine carefully before operation, measure and record vital signs, and prepare skin in operation area before operation. After operation, prevent and nurse correct postures and complications. With the development of medicine and the coming of high technology age, more and more new theory and technology appear, orthopaedics develops rapidly.

Facing this situation, nursing staff in Orthopedic Department should improve and renew professional theory and nursing technology urgently. Patients in the intervention group are treated with nursing intervention on the basis of the control group. After nursing, the rate of psychological reaction in the intervention group was much higher than that in the control group. Different results between the two groups were significant in Statistics; however, the rate of pain occurrence in the intervention group was much lower than that in the control group. Different results between the two groups were significant in Statistics. Therefore, clinical effect in the intervention group was much better than that in the control group after nursing intervention. Nursing intervention should be advocated.

\subsection{Advantages of nursing intervention}

Nursing intervention is a method to treat diseases by using nursing measures. With the change of modern medical mode, psychological nursing draws people's attention, and is widely recognized and widely used in clinical nursing practice. As an important part of modern nursing mode, psychological nursing should be used through the whole process of clinical nursing and nursing practice. It is im- portant to do well in psychological nursing and guidance, master and improve communicative skills [7-10]. Face most patients' fear, it's important for psychological nursing to reduce and eliminate the danger in order to strengthen the sense of security. Before the fear occurrence, medical staff should explain the possible pain and threat to patients and give them safety suggestion and guarantee. When patients face fear, they must be treated kindly, calmly and stable.

Every action should give patients safety suggestion and guarantee. Help patients release fear by guiding them to learn physical and mental relaxation, deep breathing and imagination of the palm fever [11]. Behavior nursing guides patients to recover function exercise in time. Recover local limb function and health in whole body. Strengthen active motions and loaded exercise of affected limb joints, which makes joints recover in normal active range and strength. Functional exercise not only emphasizes the local exercise, but also the whole body's activities to prevent and reduce the occurrence of fracture disease [12-13]. During the orthopedic behavior intervention, actions should be oriented; they must be taken step by step, not being impatient for success. Notice safety and avoid injury. Do not take exercises of increasing gravity of limbs and spinning upper and lower of the fracture, such as active knee extension under knees bending position \& straight-leg raising of leg fracture, shoulder joint abduction under erect position of femoral shaft, spinning activities of front hip fracture, and knee flexion \& extension under sitting position of femoral shaft fracture.

In a word, the author believes that nursing intervention can obviously improve patients' psychological state, reduce the occurrence of pain, and is good for the rehabilitation of patients.

\section{References}

1. Zhang Q. Prevention and treatment of lower extremity deep vein thrombosis after surgery. Chinese Journal of Practical Surgery. 2001;21(5):263-264.

2. Gao L, Ma Y, Miao J, et al. Relativity between lower limb swelling and hemorrheology after total hip arthroplasty. Chinese Journal of Nursing. 2004;39(4):247-249.

3. Xu Y, Hou D, Pan E. Effect of nursing intervention on the incidence of complications caused by intracerebral hemorrhagic. Chinese Journal of Practical Nursing. 2006;22(13):5-6.

4. Cakir O, Subasi M, Erdem K, et al. Treatment of vascular injuries associated with limb fracture. Ann $R$ Coll Surg Engl. 2005;87(5): 348-352.

5. Coats TJ, Brazil E, Hel'on M. The effects of commonly used resuscitation fluids on whole blood coagulation. Emerg Med J. 2006;23(7):546-549.

6. Jia Z, Yang J, Xing G, et al. External fixator combined with internal fixation for the treatment of distal radius comminuted fracture in 21 cases. Chinese Journal of 
Bone and Joint Injury. 2005;20(8):550.

7. Chen Q, Cheng Y, Wang J, et al. Evidence-based nursing in the bedsore nursing clinical practice. Journal of Nurses Training. 2002;17(11):846-847.

8. Su D, Fan Q, Liu G, et al. Practical pain nursing. Wuhan: Hubei publishers of science and technology. 2000;65101.

9. Wang J, Chen G, Su W, et al. Influence of nursing intervention on life style of hypertension patients in community. Chinese Nursing Research. 2007; 21(34): 3188-3189.

10. Wang S, Lin Fen, Tang H. The nursing of 2 cases of spinal cord ischemia reperfusion injury. Chinese Journal of
Nursing. 2007; 42;(9):799-800.

11. Yin X, Wang J, Hu X, et al. Effect of psychotherapy during physiotherapy in patients with cervical spondylosis. Journal of the Fourth Military Medical University. 2007;28(4):367-369.

12. Zhang F, Gan M. The application of clinical nursing path in orthopedic surgery patients. The Journal of Practical Medicine. 2007;23(14):2270-2271.

13. Tang H, Zhang L, Pi H, et al. The analysis of risk of deep vein thrombosis after the system early activities scheme reduced the joint replacement. Chinese Journal of Nursing. 2007;11(11):965-968. 